Stephan Russenschuck

\title{
Field Computation for Accelerator Magnets
}

Analytical and Numerical Methods for

Electromagnetic Design and Optimization

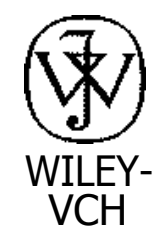

WILEY-VCH Verlag GmbH \& Co. KGaA 


\section{Contents}

Preface $\boldsymbol{X V}$

\section{Notation $X I X$}

$1 \quad$ Magnets for Accelerators 1

1.1 The Large Hadron Collider 2

$1.2 \quad$ A Magnet Metamorphosis 7

1.3 Superconductor Technology 16

1.3.1 Critical Current Density of Superconductors 16

1.3.2 Strands 19

1.3.3 Cables 22

1.4 The LHC Dipole Coldmass 27

1.5 Superfluid Helium Physics and Cryogenic Engineering 29

1.6 Cryostat Design and Cryogenic Temperature Levels 32

1.7 Vacuum Technology 33

1.8 Powering and Electrical Quality Assurance 35

1.9 Electromagnetic Design Challenges 38

1.9.1 The CERN Field Computation Program ROXIE 42

1.9.2 Analytical and Numerical Field Computation 44 References 46

$2 \quad$ Algebraic Structures and Vector Fields 49

$2.1 \quad$ Mappings 49

2.2 Groups, Rings, and Fields 50

2.3 Vector Space 51

2.3.1 Linear Independence and Basis 53

2.4 Linear Transformations 54

$2.5 \quad$ Affine Space 56

2.5.1 Coordinates 58

2.6 Inner Product Space 60 
2.6.1 Metric Space 62

2.6.2 Orthonormal Bases 63

2.6.3 The Erhard Schmidt Orthogonalization 64

2.7 Orientation 66

2.8 A Glimpse on Topological Concepts 68

2.8.1 Homotopy 69

2.8.2 The Boundary Operator 70

2.9 Exterior Products 72

2.10 Identities of Vector Algebra 75

2.11 Vector Fields 75

2.12 Phase Portraits 77

2.13 The Physical Dimension System 80

References 84

3 Classical Vector Analysis 85

3.1 Space Curves 86

3.1.1 The Frenet Frame of Space Curves 88

3.2 The Directional Derivative 93

3.3 Gradient, Divergence, and Curl 94

3.4 Identities of Vector Analysis 96

3.5 Surfaces in $E 396$

3.6 The Differential 98

3.7 Differential Operators on Scalar and Vector Fields in $\mathrm{r}$ and $\mathrm{r}^{\prime} 102$

3.8 The Path Integral of a Vector Field 103

3.9 Coordinate-Free Definitions of the Differential Operators 104

3.10 Integral Theorems 106

3.10.1 The Kelvin-Stokes Theorem 106

3.10.2 Green's Theorem in the Plane 107

3.10.3 The Gauss-Ostrogradski Divergence Theorem 108

3.10.4 A Variant of the Gauss Theorem 108

3.10.5 Green's First Identity 109

3.10.6 Green's Second Identity (Green's Theorem) 110

3.10.7 Vector Form of Green's Theorem 110

3.10.8 Generalization of the Integration-by-Parts Rule 110

3.10.9 The Stratton Theorems 111

3.11 Curvilinear Coordinates 111

3.11.1 Components of a Vector Field 113

3.11.2 Contravariant Coefficients 114

3.11.3 Covariant Coefficients 115

3.12 Integration on Space Elements 115

3.13 Orthogonal Coordinate Systems 117

3.13.1 Differential Operators 119 
3.13.2 Cylindrical Coordinates 121

3.13.3 Spherical Coordinates 122

3.14 The Lemmata of Poincare 125

3.15 De Rham Cohomology 126

3.16 Fourier Series 129

References 136

4 Maxwell's Equations and Boundary Value Problems in Magnetostatics 137

4.1 Maxwell's Equations 138

4.1.1 The Global Form 138

4.1.2 The Integral Form 139

4.1.3 The Local Form 141

4.1.4 Maxwell's Original Set of Equations 142

4.2 Kirchhoff's Laws 143

4.3 Conversion of Energy in Electromagnetic Fields 143

4.4 Constitutive Equations 144

4.5 Boundary and Interface Conditions 146

4.6 Magnetic Material 151

4.6.1 Ferromagnetism 152

4.6.2 Measurement of Hysteresis Curves 155

4.6.3 Magnetic Anisotropy in Laminated Iron Yokes 159

4.6.4 Magnetostriction 160

4.6.5 Permanent Magnets 161

4.6.6 Magnetization Currents and Fictitious Magnetic Charges 163

4.7 Classification Diagrams for Electromagnetism 165

4.8 Field Lines 167

4.8.1 Classification of Electromagnetic Field Problems 167

4.9 Boundary Value Problems 1: Magnetostatic 171

4.9.1 Scalar-Potential Formulations 171

4.9.2 Vector-Potential Formulations 173

4.9.3 The Scalar Laplace Equation in 2D 179

4.10 Boundary Value Problems 2: Magnetic Diffusion Problems 180

References 184

$5 \quad$ Fields and Potentials of Line_Currents 187

5.1 Green Functions 188

5.2 Potentials an Bounded Domains 189

5.3 Properties of Harmonic Fields 191

5.4 The Biot-Savart Law 193

5.5 Field of a Straight Line-Current Segment 197

5.6 Field of a Ring Current 200 
5.7 The Magnetic Dipole Moment 203

5.8 The Magnetic Double Layer 205

5.8.1 The Solid Angle 206

5.8.2 Approximating the Solid Angle of a Current Loop 208

5.9 The Image-Current Method 209

5.9.1 Plane Boundaries 211

5.9.2 Circular Boundaries 213

5.10 Stored Energy in a Magnetostatic Field 216

5.10.1 Self and Mutual Inductance 218

5.10.2 The Geometric Mean Distance 220

5.10.3 Magnetic Flux 222

5.11 Magnetic Energy in Nonlinear Circuits 224

5.11.1 Differential Inductance 224

5.12 Magnetic Forces and the Maxwell Stress Tensor 227

5.13 Fields and Potentials of Magnetization Currents 230

5.14 Magnetic Levitation 232

References 235

$6 \quad$ Field Harmonics 237

6.1 Circular Harmonics 238

6.1.1 Determining the Multipole Coefficients 240

6.1.2 Magnetic Shielding; Permeable Cylindrical Shell in a Uniform Field 253

6.1.3 Integrated Multipoles in Accelerator Magnets 255

6.2 Spherical Harmonics 257

6.2.1 Legendre Series Expression for the Vector Potential 262

6.2.2 Determining the Zonal Harmonics 263

6.3 Separation in Cartesian Coordinates 265

References 268

7 Iron -Dominated Magnets 269

7.1 C-Shaped Dipole 270

7.2 Quadrupole 271

7.3 Ohmic Losses in Dipole and Quadrupole Coils 272

7.4 Magnetic Circuit with Varying Yoke Width 272

7.5 Branched Circuits 274

7.6 Ideal Pole Shapes of Iron-Dominated Magnets 275

7.6.1 Shimming 277

7.7 Rogowski Profiles 278

7.8 Combined-Function Magnets 281

7.9 Permanent Magnet Excitation 282

7.10 Cooling of Normal-Conducting Magnets 287

References 291 


\section{Coil-Dominated Magnets 293}

8.1 Accelerator Magnets 294

8.1.1 Generation of Pure Multipole Fields 295

8.1.2 Sensitivity to Coil-Block Positioning Errors 305

8.1.3 Force Distribution 305

8.1.4 Margins in the LHC Main Dipole 306

8.2 Combined-Function Magnets and the Unipolar Current Dipole 309

8.3 Rectangular Block-Coil Structures 310

8.4 Field Enhancement in Coil Ends of Accelerator Magnets 311

8.5 Magnetic Force Distribution in the LHC Dipole Coil Ends 312

8.6 Nested Helices 314

8.7 Solenoids 315

8.7.1 Helmholtz and Maxwell Coils 315

8.7.2 Fabry Factors 317

8.7.3 Off-Axis Fields 321

8.7.4 Zonal Harmonics 324

References 325

9 Complex Analysis Methods for Magnet Design 327

9.1 The Field of Complex Numbers 328

9.2 Holomorphic Functions and the Cauchy-Riemann Equations 329

9.3 Power Series 331

9.4 The Complex Form of the Discrete Fourier Transform 333

9.5 Complex Potentials 335

9.6 Conformal Mappings 336

9.7 Complex Representation of Field Quality in Accelerator Magnets 338

9.7.1 Feed-Down 338

9.7.2 Reference Frame Rotation 342

9.7.3 Reflection about the Vertical Axis 343

9.8 Complex Integration 344

9.8.1 Cauchy's Theorem and the Integral Formula 345

9.8.2 Properties of Holomorphic Functions 346

9.8.3 The Residual Theorem 348

9.9 The Field and Potential of a Line Current 349

9.9.1 Series Expansion of the Line-Current Field 350

9.9.2 Circular Sector Windings 351

9.10 Multipoles Generated by a Magnetic Dipole Moment 351

9.11 Beth's Current-Sheet Theorem 352

9.12 Electromagnetic Forces an the Dipole Coil 354

9.13 The Field of a Polygonal Conductor 356

9.14 Magnetic Flux Density Inside Elliptical Conductors 358 References 362 
$10 \quad$ Field Diffusion 363

10.1 Time Constants and Penetration Depths 363

10.2 The Laplace Transform 365

10.3 Conductive Slab in a Time-Transient Applied Field 370

10.3.1 The Step-Excitation Function 371

10.3.2 Linear Ramp of the Applied Field 373

10.3.3 Sinusoidal Excitation 375

10.4 Eddy Currents in the LHC Cold Bore and Beam Screen 376 References 382

$11 \quad$ Elementary Beam Optics and Field Requirements 383

11.1 The Equations of Charged Particle Motion in a Magnetic Field 383

11.2 Magnetic Rigidity and the Bending Magnets 387

11.3 The Linear Equations of Motion 389

11.4 Weak Focusing 390

11.5 Thin-Lens Approximations 392

11.6 Transfer Matrices 393

11.7 Strong Focusing and the FODO Cell 395

11.8 The Beta Function, Tune, and Transverse Resonances ; 397

11.9 Off-Momentum Particles 407

11.9.1 Dispersion 408

11.9.2 Chromaticity 410

$11.10 \quad$ Field Error Specifications 412

References 413

12 Reference Frames and Magnet Polarities 415

12.1 Magnet Polarity Conventions 416

12.1.1 Spool-Piece Correctors 418

12.1.2 Twin-Aperture and Two-in-One Magnets 418

12.2 Reference Frames 420

12.3 Multipole Expansions 421

12.3.1 The Magnet Frame 421

12.3.2 The Local Reference Frame of Beam 1423

12.3.3 Definition of Field Errors in the Accelerator Design Program MAD 424

12.3.4 Transformation between the Magnet and the Beam 1 Frames 424

12.4 Orbit Correctors 426

12.5 Position of the Connection Terminals 426

12.6 Turned Magnets and Magnet Assemblies 427

12.7 Electrical Circuits in the LHC Machine 429

References 432 
$13 \quad$ Finite-Element Formulations 433

13.1 One-Dimensional Finite-Element Analysis 434

13.1.1 Quadratic Elements 439

13.2 FEM with the Vector-Potential (Curl-Curl) Formulation 441

13.2.1 The Weak Form in 3D 443

13.2.2 The Weak Form in 2D 444

13.3 Complementary Formulations 445

13.3.1 FEM with Reduced Vector-Potential Formulation 445

13.3.2 FEM, Employing the Vector Poisson Equation 449

13.3.3 The A-0 Formulation for Eddy-Current Problems 451 References 453

14 Discretization 455

14.1 Quadrilateral Mesh Generation 456

14.1.1 Parametric Modeling 457

14.1.2 Topology Decomposition 458

14.1.3 Domain Decomposition 459

14.1.4 Meshing of Simple Domains 460

14.1.5 Smoothing 461

14.1.6 Remeshing and Morphing 462

14.2 Finite-Element Shape Functions 465

14.2.1 The Linear Triangular Element, 2D 466

14.2.2 Barycentric Coordinates 469

14.2.3 Local Coordinates 470

14.2.4 Mapped Elements 471

14.2.5 Generation of the Shape Functions 474

14.2.6 Transformation of Differential Operators 476

References 479

15 Coupling of Boundary and Finite Elements 481

15.1 The Boundary-Element Method 482

15.1.1 The Node Collocation Method 486

15.2 BEM-FEM Coupling 487

15.3 BEM-FEM Coupling using the Total Scalar-Potential 489

15.4 The M(B) Iteration 491

15.5 Applications 492

15.5.1 2D Calculations 492

15.5.2 Saturation Effects in the Iron Yoke 495

15.5.3 3D Calculations 496

References 502 
16 Superconductor Magnetization 503

16.1 Superconductor Magnetization 507

16.2 Critical Surface Modeling 509

16.3 The Critical State Model 513

16.4 The Ellipse an a Cylinder Model 516

16.5 Nested Intersecting Circles and Ellipses 519

16.6 Hysteresis Modeling 521

16.7 Magnet Field Errors due to the Superconducting Filament Magnetization 527

16.8 The $\mathrm{M}(\mathrm{B})$ Iteration 530

16.9 Software Implementation 532

16.10 Applications to Magnet Design 532

16.10.1 Compensation of Multipole Field Errors 534

$16.11 \quad$ Nested Magnets 537

References 540

$17 \quad$ Interstrand Coupling Currents 543

17.1 Analysis of Linear Networks 544

17.1.1 The Linear U(I) Relation in a Branch 545

17.1.2 The Topology of Networks 547

17.1.3 The Branch/Node Incidence Matrix and the Node-Potential Method 548

17.1.4 The Mesh Matrix and the Mesh-Current Method 551

17.1.5 Transient Field Analysis 553

17.2 A Network Model for the Interstrand Coupling Currents 555

17.3 Steady-State Calculations 557

17.3.1 Spectral Analysis of the Solution 559

17.4 Time-Transient Analysis 560

17.4.1 Spectral Analysis of the Solution 561

17.5 The M(B) Iteration Scheme for ISCCs 562

17.6 Approximation for the Interstrand Coupling Currents 563

17.7 Interfilament Coupling Currents 564

17.8 Applications to Magnet Design 566

17.8.1 Field Advance 566

17.8.2 Rapid Cycling Magnets 567

References 572

18 Quench Simulation 575

18.1 The Heat Balance Equation 577

18.2 Electrical Network Models of Superconductors 580

18.3 Current Sharing 582

18.4 Winding Schemes and Equivalent Electrical Circuit Diagrams 584 
18.5 Quench Detection 585

18.6 Magnet Protection 586

18.7 Numerical Quench Simulation 589

18.7.1 The Thermal Model 591

18.7.2 External Electrical Circuits 594

18.8 The Time-Stepping Algorithm 595

18.9 Applications 596

18.9.1 Validating the Model 598

18.9.2 Fast Ramping Magnets 601

References 607

19 Differential Geometry Applied to Coil_End Design 609

19.1 Constant-Perimeter Coil Ends 612

19.2 Differential Geometry of the Strip Surfaces 615

19.2.1 The Frenet-Serret Equations for Strips 616

19.2.2 The Generators of Strips 618

19.3 Discrete Theory of the Strip Surface 621

19.4 Optimization of the Strip Surface 627

19.5 Coil-End Transformations 630

19.6 Corrector Magnet Coil End with Ribbon Cables 631

19.7 End-Spacer Manufacturing 633

19.8 Splice Configurations 634

References 636

$20 \quad$ Mathematical Optimization Techniques 637

20.1 Mathematical Formulation of the Optimization Problem 639

20.2 Optimality Criteria for Unconstrained Problems 641.

20.3 Karush-Kuhn-Tucker Conditions 642

20.4 Pareto Optimality 644

20.5 Methods for Decision Making 646

20.5.1 Goal Programming 646

20.5.2 The Pareto-Strength Algorithm 650

20.5.3 Constraint Formulation and Sensitivity Analysis 651

20.5.4 Payoff Tables 653

20.6 Box Constraints 654

20.7 Treatment of Nonlinear Constraints 655

20.8 Deterministic Optimization Algorithms 655

20.8.1 Line Search 656

20.8.2 Multidimensional Search Methods 659

20.8.3 Gradient Methods 660

20.9 Genetic Optimization Algorithms 667

20.9.1 Parameter Representation 669 
20.9.2 Gray Coding 669

20.9.3 Genetic Operators 671

20.9.4 Convergence 678

20.10 Applications 681

20.10.1 Conceptual Coil Design with Genetic Algorithms 683

20.10.2 Deterministic Optimization of Coil Cross Sections 685

20.10.3 Yoke Design as a Material-Distribution Problem 688

20.10.4 Shape Optimization of the Iron Yoke 689

20.10.5 Payoff Tables for Dipole Designs 691

20.10.6 Lagrange Multiplier Estimation 693

20.10.7 Manufacturing Tolerances 694

20.10.8 Tuning Range 696

20.10.9 Tracing of Manufacturing Errors 697

References 700

\section{Appendix}

A Material Property Data for Quench Simulations 703

A.1 Mass Density 703

A.2 Electrical Resistivity 705

A.3 Thermal Conductivity 708

A.4 Heat Capacity 711

References 715

B The LHC Magnet Zoo 717

B.1 Superconducting Magnets 717

B.2 Normal-Conducting Magnets 726

References 729

C Ramping the LHC Dipoles 731

D $\quad$ SI (MKSA) Units 735

E Glossary 737

Index 745 